\title{
Tendencias de infecciones intrahospitalarias en un centro oncológico, 1986-1996
}

\author{
Patricia Volkow, M.C., ${ }^{(1)}$ Margarita de la Rosa, Lic. en Enf., ${ }^{(1)}$ Patricia Gordillo, Lic. en Enf., (1) \\ Diana Vilar-C ompte, M.C., M.SC., (1) Sergio Lazo de la Vega, M.C., (1) Grisel A randa-C ortés, Lic. en Enf., (1) \\ Silvia Sandoval, Lic. en Enf. ${ }^{(1)}$
}

\begin{abstract}
Volkow P, De la Rosa M, Gordillo P, Vilar-Compte D, Lazo de la Vega S, A randa-Cortés G, Sandoval S. Tendencias de infecciones intrahospitalarias en un centro oncológico, 1986-1996. Salud Publica Mex 2000;42:181-187.
\end{abstract}

\begin{abstract}
Resumen
Objetivo. Describir los resultados de diez años de vigilancia de infecciones intrahospitalarias (II) en un centro oncológico. Material y métodos. Es un estudio descriptivo, retrospectivo, del programa de vigilancia del Comité de Vigilancia y Control de Infecciones Intrahospitalarias del Instituto Nacional de Cancerología, realizado en 1997. Se utilizaron los criterios de infección intrahospitalaria por sitio propuestos por el Centro para la Prevención y el Control de Enfermedades deA tlanta, $G$ eorgia, de Estados Unidos de América, en 1972. De junio de 1986 a diciembre de 1996, se estudiaron 62733 egresos. La vigilancia se realizó mediante la revisión de los expedientes de pacientes con fiebre, de aquellos que reciben antibióticos y de posoperados en los pisos de hospitalización y revisión de cultivos. Se calcularon las tasas de II por episodios/100 egresos. Resultados. La tasa de infecciones intrahospitalarias por 100 egresos fue de 4.4 en 1986; 7.7, en 1987; 8.1, en 1988; 5.9, en 1989; 4.6 , en 1990; 5.1 , en 1991; 4.3, en 1992; 5.4, en 1993; 7.6, en $1994 ; 7.1$, en 1995, y 8.5, en 1996. El germen más frecuentemente aislado en las II fue Escherichia coli. Los aislamientos de hongos y los de enterococo se incrementaron en casi siete veces desde 1987. Conclusiones. Se observó un incremento en la tasa de infecciones hospitalarias en los últimos cuatro años. Al menos dos factores contribuyeron a esta tendencia: la aplicación de un sistema de vigilancia más experimentado, el cual hizo que disminuyera el subregistro, y el aumento real en la frecuencia de tales infecciones.
\end{abstract}

Palabras clave: infección hospitalaria/tendencias; instituciones oncológicas; México
Volkow P, De la Rosa M, Gordillo P, Vilar-Compte D, Lazo de la Vega S, A randa-Cortés G, Sandoval S.

Trends in nosocomial infections

in an oncology center, 1986-1996.

Salud Publica Mex 2000;42:181-187.

\begin{abstract}
A bstract
Objective To describe the results of ten years of nosocomial infection (NI) surveillance in an oncology center. Material and methods This is a descriptive study of the Infection Control and Surveillance Program Committee at the Instituto $\mathrm{N}$ acional de $\mathrm{C}$ ancerología, conducted in 1997. From June 1986 to December 1996, we surveyed 62733 hospital discharge records. Criteria used to classify nosocomial infections were those outlined in 1972 by the Centers for Disease Control and Prevention, Atlanta (GA). Survey data were collected through review of microbiology chart records and of hospital chart records of febrile patients, patients receiving antibiotics, and patients visited after surgery.W e calculated the rates of $\mathrm{N} I$ as the number of infections/100 discharges. Results. The rate of NI per 100 discharges was 4.4 in 1986, 7.7 in 1987, 8.1 in 1988, 5.9 in $1989,4.6$ in 1990, 5.1 in 1991, 4.3 in 1992, 5.4 in 1993, 7.6 in $1994,7.1$ in 1995 and 8.5 in 1996. Escherichia coli was the microorganism most frequently isolated. From 1987, an increase of almost seven times in fungi isolations as well as enterococci was obser ved.Conclusions $A n$ increasing trend in $\mathrm{NI}$ rates was observed in the last four years, probably related to multiple factors such as improved surveillance (better reporting) and a real increase in the frequency of N I.
\end{abstract}

Key words: nosocomial infection/trends; cancer care facilities; Mexico

(1) Departamento de Infectología, Instituto $\mathrm{N}$ acional de Cancerología, México, D.F., México.

Fecha de recibido: 9 de agosto de 1999 - Fecha de aprobado: 21 de febrero de 2000

Solicitud de sobretiros: D ra. Patricia Volkow. Departamento de Infectología, Instituto N acional de Cancerología. San Fernando 22, colonia Tlalpan, 14000 México, D.F., México. Correo electrónico: volkow@ infosel.net.mx 
$\mathrm{E}$ l cáncer se ha convertido en la segunda causa de muerte en México ${ }^{1} y$, por tanto, constituye un grave problema de salud pública. Esta situación ha incrementado la demanda de atención médica especializada por parte de los pacientes que sufren la enfermedad. Durante las tres últimas décadas se han logrado avances en el tratamiento del cáncer como: a) nuevos agentes de quimioterapia; b) esquemas combinados más eficaces, pero a su vez más tóxicos y c) trasplantes de médula ósea y esquemas con altas dosis de quimioterapia con rescate de células progenitoras de sangre periférica, etcétera. Asimismo, se han ampliado los alcances de sofisticadas y extensas intervenciones quirúrgicas acompañadas de reconstrucciones fisiológicas que permiten mejorar el pronóstico de los pacientes oncológicos. Sin embargo, todas estas aplicaciones e intervenciones aumentan los riesgos de que los pacientes sufran infecciones intrahospitalarias. $^{2-4}$ En las dos últimas décadas las infecciones se han convertido en la primera causa de muerte de los enfermos con cáncer, especialmente aquellos con neutropenia prolongada. 5

En el presente estudio se presentan los resultados de diez años de vigilancia de infecciones intrahospitalarias (II), así como el impacto que los diferentes programas de prevención y vigilancia han producido durante el periodo.

\section{Material y métodos}

Es un estudio descriptivo, retrospectivo, del Programa del Comité de Vigilancia y Control de Infecciones Intrahospitalarias del Instituto Nacional de Cancerología (INCan), realizado en 1997. Se definió como infección intrahospitalaria, aquella que no estaba presente o incubándose al momento del internamiento y se inicia después de 48 a 72 horas del ingreso al hospital. Los criterios para clasificar un episodio de infección intrahospitalaria por sitio fueron tomados de los lineamientos dictados por el Centro para la Prevención y Control de Enfermedades de Atlanta, Georgia, de los Estados Unidos de América (EUA). ${ }^{6}$

El INCan es un hospital de enseñanza, de tercer nivel; recibe pacientes adultos con cáncer, para ello cuenta con 117 camas de hospitalización, distribuidas en tres pisos: el de Oncología Médica, el de Cirugía Oncológica y el de Radioterapia. Cuenta además con una Unidad de Cuidados Intensivos con seis camas y, a partir de junio de 1991, con una Unidad de Transplante de Médula Osea con tres camas de aislamiento total y dos de aislamiento parcial. En promedio se registraron 7000 egresos anuales en los últimos cinco años. El Comité de Vigilancia y Control de Infecciones Intrahospitalarias inició su trabajo en $1986 .{ }^{7}$

Dicho comité contó con una enfermera hasta 1987; con dos enfermeras, hasta 1993, y actualmente hay tres enfermeras dedicadas exclusivamente a ejercer la vigilancia. Esta se realiza de lunes a viernes mediante visitas programadas a las áreas de hospitalización en donde se revisan los expedientes de pacientes con fiebre, o que reciben antibióticos y se examinan los pacientes posoperados. A los pacientes con sonda urinaria se les toma urocultivo al momento de instalación y el proceso se repite cada cinco días y al retiro de la sonda. Con la misma frecuencia se revisan las libretas del laboratorio de microbiología; allí se recaban los resultados de hemocultivos, urocultivos y cultivos diversos, y se revisan todos los expedientes de pacientes con cultivos positivos.

Durante estos diez años se implementaron diversos programas: en agosto de 1988, se incorporó el equipo de terapia intravenosa (ETI) para cuidado de vías vasculares de larga estancia; en agosto de 1991, se extendió el cuidado del ETI a todos los catéteres centrales de estancia intrahospitalaria; en febrero de 1992, se inició en forma intermitente el Programa Prospectivo de Vigilancia de Herida Quirúrgica (HQX) y, de manera permanente a partir de 1993. Este programa consiste en abrir al día siguiente del procedimiento quirúrgico una hoja de seguimiento para cada cirugía realizada en el hospital, en la que se recaban los siguientes datos: nombre, número de expediente, edad, enfermedad de base, procedimiento quirúrgico (si fue electivo o de urgencias), clasificación por el tipo de cirugía, tiempo de permanencia de los drenajes, tiempo de estancia pre y posoperatoria y seguimiento durante 30 días, utilizando para ello el expediente clínico y los resultados de cultivos de laboratorio de microbiología.

Se calculó la tasa de II general por episodios/100 egresos hospitalarios para cada año de 1995 a 1996 (antes de 1994 se excluyeron de la vigilancia de infecciones intrahospitalarias las áreas de radioterapia y braquiterapia, que representaba $10 \%$ de los egresos hospitalarios); de 1986 a 1994 el denominador fueron únicamente los egresos vigilados. Los sitios de infección se expresan en porcentajes del total anual. También se calculó la tasa de II por sitio de infección por episodio/100 egresos, utilizando el número de eventos por sitio de infección como numerador. La tasa de infección de HQX/100 cirugías se calculó utilizando como denominador las cirugías mayores realizadas en cada año (las cirugías menores rara vez se hospitalizan y por ello, hasta antes del Programa Prospectivo 
de Vigilancia de Herida Quirúrgica, no eran vigiladas). A partir de 1993 la tasa de las infecciones de herida quirúrgica está calculada por medio del programa de vigilancia prospectiva que cubre el total de las cirugías realizadas.

Para evaluar el posible impacto de los diferentes programas del Comité de Vigilancia y Control de Infecciones Intrahospitalarias se comparó la prevalencia de infecciones intrahospitalarias entre dos diferentes periodos: antes y después del inicio del programa preventivo o sistema de vigilancia.

Se calculó el riesgo relativo y se estimaron intervalos de confianza a 95\% (IC 95\%). El estadístico de prueba fue $\chi^{2}$. Se consideró estadísticamente significativa una $p \leq 0.05$.

\section{Resultados}

De junio de 1986 a diciembre de 1996 se vigilaron 62733 egresos. La tasa de II por 100 egresos fue de 4.4 en 1986; 7.7, en 1987; 8.1, en 1988; 5.9 , en $1989 ; 4.6$, en 1990; 5.1, en 1991; 4.3, en 1992; 5.4, en 1993; 7.6, en 1994; 7.1, en 1995, y 8.5, en 1996. En la figura 1 se presenta la tasa de II general por año, así como el número de egresos, y el promedio de días de estancia hospitalaria.

El número de cirugías realizado antes de instaurar el programa de vigilancia fue de 17759 y después de su puesta en marcha, de 11 488, observándose un

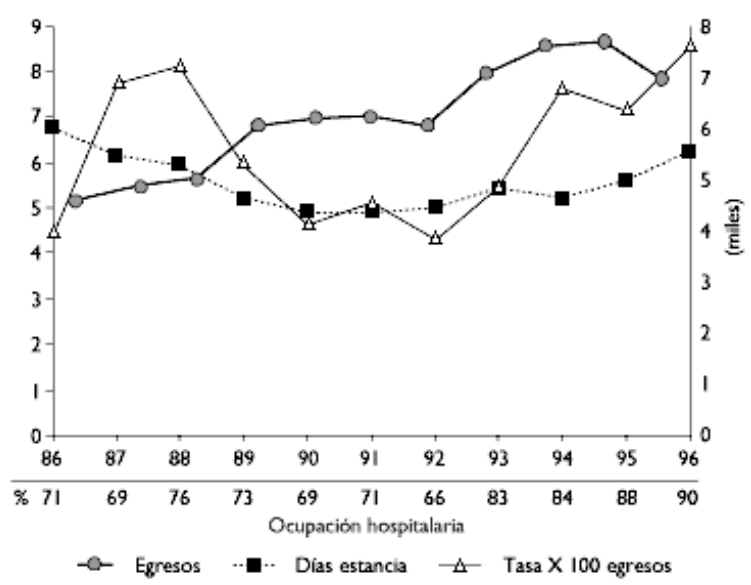

Figura 1.TASA ANUAL de INFECCIÓN INTRAHOSPITALARIA POR 100 EGRESOS, PROMEDIO DE DÍAS ESTANCIA POR PACIENTE Y NÚMERO DE EGRESOS HOSPITALARIOS. INStituto Nacional de Cancerología, México, 19861996 incremento de $13 \%$ en el número de cirugías realizadas en el segundo periodo. Durante estos 10 años no aumentó el número de plazas de la plantilla de enfermería del área de hospitalización ni se incrementó el número de quirófanos. El cuadro I muestra la tasa anual de infección por sitio por 100 egresos, la infección de HQX está calculada por 100 cirugías mayores realizadas cada año.

La figura 2 muestra la distribución de infecciones intrahospitalarias por sitio. Destaca el incremento de infección intrahospitalaria de heridas quirúrgicas a partir de 1992, que coincide con el inicio del Programa Prospectivo de Vigilancia de Herida Quirúrgica de manera intermitente. De 1986 a 1991, ocurrieron 429 episodios de infección de herida quirúrgica, con una tasa de 2.4 por 100 cirugías mayores realizadas; en el periodo de 1992 a 1996 se registraron 937 episodios de infección de herida quirúrgica con una tasa de 8.5 por 100 cirugías mayores realizadas $(p<0.001)$.

En la figura 3 se registran las distribuciones porcentuales de los gérmenes identificados en infecciones intrahospitalarias. En ésta se aprecia el porcentaje que representan los hongos en la distribución de los gérmenes identificados. La gráfica no ofrece datos por especie, ya que la infraestructura de laboratorio para identificar los diferentes hongos ha cambiado en el tiempo y no es posible unificar la información. Durante los primeros años se identificaban únicamente como

\section{Cuadro 1}

TASA ANUAL DE INFECCIÓN INTRAHOSPITALARIA, POR SITIO, POR 100 EGRESOS

\begin{tabular}{llllcl} 
Año & IVU & IHQX* & Neumonía & Bacteriemia 1 & Bacteriemia 2a \\
1986 & 1.34 & 2.9 & 0.46 & 0.12 & 0.12 \\
\hline 1987 & 2.98 & 3.13 & 0.9 & 0.78 & 0.40 \\
\hline 1988 & 2.69 & 3.83 & 0.5 & 1.46 & 0.58 \\
\hline 1989 & 2.35 & 3.94 & 0.53 & 0.92 & 0.16 \\
\hline 1990 & 1.53 & 3.33 & 0.49 & 0.74 & 0.11 \\
\hline 1991 & 1.48 & 5.67 & 0.55 & 0.52 & 0.07 \\
\hline 1992 & 1.143 & 4.97 & 0.38 & 0.57 & 0.13 \\
\hline 1993 & 1.12 & 9.79 & 0.47 & 0.41 & 0.13 \\
\hline 1994 & 1.46 & 10.97 & 0.95 & 1.32 & 0.25 \\
\hline 1995 & 1.75 & 8.07 & 1.03 & 1.02 & 0.26 \\
\hline 1996 & 1.48 & 9.07 & 1.53 & 1.16 & 0.25
\end{tabular}

* La infección de herida quirúrgica está calculada por episodios de infección de herida quirúrgica/100 cirugías mayores realizadas. Instituto $\mathrm{N}$ acional de Cancerología, México, 1986-1996

IVU = infección de vías urinarias

$I H Q X=$ infección de herida quirúrgica 


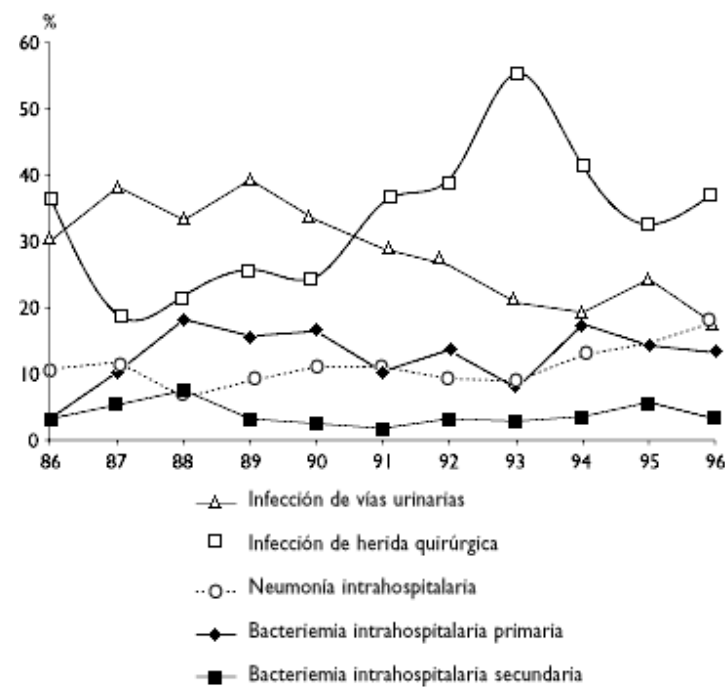

Figura 2. Distribución porcentual de infecciones INTRAHOSPITALARIAS POR SITIO DE INFECCIÓN, POR AÑO de vigilancia. Instituto Nacional de Cancerología, MéxıICo, 1986-1996

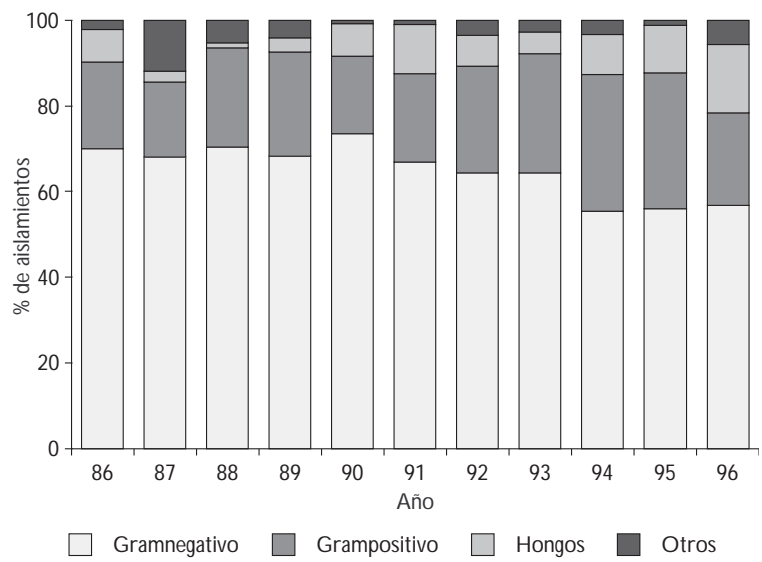

Figura 3. Distribución porcentual de gérmenes GRAMPOSITIVOS, GRAMNEGATIVOS Y HONGOS IDENTIFICADOS EN INFECCIONES INTRAHOSPITALARIAS. INSTITUTO Nacional de Cancerología, México, 1986-1996

levaduras o Cándida sp; posteriormente fue posible determinar la especie. En la figura 4 se presentan los porcentajes de bacterias grampositivas y gramnegativas.
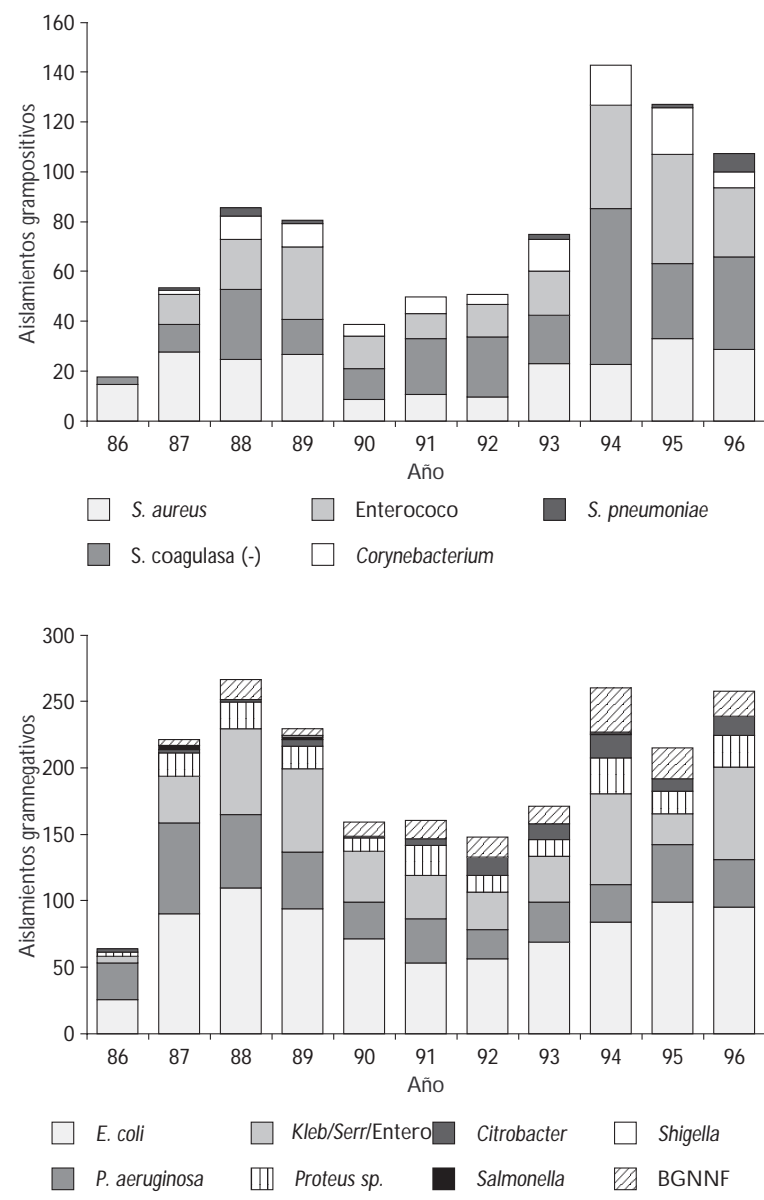

Figura 4. Porcentaje de gérmenes grampositivos, GRAMNEGATIVOS AISLADOS DE INFECCIONES INTRAHOSpitalarias por año. Instituto Nacional de Cancerología, México, 1986-1996

S AUREUS= STAPHYLOCOCCUS AUREUS, $\mathbf{S}$ COAGULASA $(-)=$ Staphylo coccus coagulasa negativa, Enterococo= Entero co c CUS SP, Strepto cocos SP. $=$ Strepto co c CUS SP. Corynebacterium=Corynebacterium SP., S. PNeUmoniAE $=$ Strepto Co CCUS PenUm O NIAE

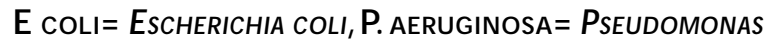
aerugino Sa, Kleb/Serr/EN = tribu Klebsiella, Serratia y Enterobacter, Proteus= Proteus SP., BGNNF= baclLos gramnegativos no-fermentadores, Citrobacter= Citro bacter sp., Salmonella= Salm onella SP., ShigelLA $=$ SHIGELLA SP

\section{Discusión}

Las complicaciones infecciosas que sufren los pacientes hospitalizados constituyen un grave problema de 
salud pública. Según Haley y colaboradores, ${ }^{8}$ anualmente en los EUA puede haber tantas infecciones como hospitalizaciones por cáncer o accidentes, y tal vez cuatro veces más infecciones intrahospitalarias que admisiones a los hospitales por infarto agudo al miocardio.

En México, el panorama no es diferente. Ponce de León informó, en 1991, ${ }^{9}$ que probablemente la razón promedio de las infecciones intrahospitalarias en los hospitales mexicanos es de $15 \%$. Si se toma en cuenta que en el país se hospitalizan tres millones de pacientes por año en el sector salud, habrá en promedio un estimado de 450000 episodios de infección intrahospitalaria anuales; si además se calcula una mortalidad asociada de $15 \%$, es posible que cada año ocurran 22500 fallecimientos relacionados con aquéllas.

En México existe reducida información sobre II en pacientes con cáncer., ${ }^{710}$ Asimismo, se cuenta con pocos informes de este tipo en la literatura anglosajona. Sin embargo, generalmente se reconoce que las tasas en los centros oncológicos son mayores a las notificadas en los hospitales generales. ${ }^{411-13}$ En los estudios de mortalidad asociada con II, $20 \%$ de los pacientes tienen una neoplasia como enfermedad de base. ${ }^{14}$ El paciente con cáncer per se sufre un mayor riesgo de infecciones por el estado de inmunocompromiso. ${ }^{15}$ A esta situación es necesario agregar que en los procedimientos de cirugía oncológica (grandes resecciones de tumores necrosados, tiempos quirúrgicos prolongados) se incrementa el riesgo de que los pacientes sufran infecciones de herida quirúrgica y otros tipos de $\mathrm{II}^{4}$ como lo informa Rotstein. ${ }^{11}$ Hallazgos similares hemos realizado en nuestra institución donde se practican más de 3000 cirugías al año. ${ }^{16}$

En esta serie se observa un incremento significativo en la tasa de infecciones intrahospitalarias, más aparente desde 1994, que de acuerdo con la información de la literatura sobre el tema resulta diferente a lo esperado. Esta situación se debe a que un sistema de vigilancia permanente tiende a disminuir las tasas de II. ${ }^{17}$ Sin embargo, variados factores, además de subregistro, así como un incremento real, pueden intervenir en esta tendencia hacia el alza de la tasa de II. Uno de estos factores podría ser la carga de trabajo. En la figura 1 destaca un incremento en los egresos hospitalarios a partir de 1993. Para evaluar la carga de trabajo se comparó el número de egresos hospitalarios en dos periodos: el primer periodo de 1986 a 1992 y el segundo periodo de 1993 a 1996. En el primer periodo (78 meses) se registraron 36741 pacientes hospitalizados (55.7\%) y 1890 II en 1531 pacientes. El promedio de estancia hospitalaria en este periodo fue de 5.5 días, y el porcentaje de ocupación hospitalaria de $69 \%$. En el segundo periodo, de 1993 a 1996 (48 meses), se registraron 29182 pacientes hospitalizados (44.3\%), y se detectó un total de 2085 episodios de II en 1667 pacientes. El promedio de estancia hospitalaria en este periodo fue de 5.6 días y el porcentaje de ocupación hospitalaria 86\%. La tasa de II de 1993 a 1996 es significativamente mayor que la de 1986 a $1992(p<0.0001)$. El número promedio de egresos hospitalarios por mes, comparando los dos periodos, mostró un incremento de $30 \%$ durante el segundo periodo, así como un aumento en el porcentaje de ocupación hospitalaria de $25 \%$.

El incremento en la tasa de II observada a partir de 1993 coincide con un incremento significativo de los egresos hospitalarios y del porcentaje de ocupación hospitalaria. Durante estos dos periodos no hubo ningún aumento de la infraestructura hospitalaria ni del número de enfermeras/cama. Este fenómeno significa un aumento real en la carga de trabajo para el personal de enfermería. El factor descrito indica cierta influencia, puesto que una mayor carga de trabajo tiende a favorecer que no se cumplan adecuadamente las medidas para la prevención de II.

Otro factor que pudo contribuir al incremento en la tasa de II, se localiza en la implantación de un mejor sistema de vigilancia que haga disminuir el subregistro, ya que por una parte el número de enfermeras que realizan la vigilancia elevó la relación enfermera/cama de 1:117 durante 1986 a 1988, a 1:58 durante 1989 a julio de 1993 y a 1:39 durante agosto de 1993 a la fecha. A estas circunstancias se agregó la implementación del Programa Prospectivo de Vigilancia de Herida Quirúrgica, el cual aumentó la captación de los episodios de infección de herida en $400 \%$ a partir de su instrumentación. $^{18}$

La implementación de un programa que centraba el cuidado de catéteres venosos centrales hospitalarios en el equipo de terapia intravenosa desde el año de 1991 no parece haber tenido impacto en la prevención de las bacteriemias intrahospitalarias primarias. Probablemente el fenómeno se deba a que estas bacteriemias no están exclusivamente relacionadas con las vías vasculares centrales y su origen se localice en la propia flora por permeación bacteriana en los pacientes neutropénicos. Otra causa alterna podría ser la contaminación de la terapia intravascular a otros niveles. No contamos con el dato de prevalencia de neutropenia en los pacientes hospitalizados. Sin embargo, el inicio del programa de transplante de médula ósea en diversas modalidades se inició en 1994.

Escherichia coli es el germen más frecuentemente aislado de las infecciones intrahospitalarias, tal como lo indica la literatura especializada. ${ }^{13,19,20}$ Los ais- 
lamientos de Pseudomonas aeruginosa disminuyeron de manera más aparente en los primeros tres años. Desde finales de los ochenta, en el hospital se hizo rutinario el uso de cefalosporinas de tercera generación con actividad anti-Pseudomonas. Ha sobrevenido un incremento en los aislamientos de Enterococo y Estafilococo coagulasa negativo como causantes de infección intrahospitalaria, al igual que los aislamientos de hongos., . $^{3,21}$ Este dato es semejante a la información que contiene la literatura anglosajona, ${ }^{22}$ ya que hospitales como el nuestro han importado la nueva tecnología para el manejo del cáncer y con ello las complicaciones que lo acompañan. También cuenta el uso generalizado de las cefalosporinas de tercera generación que favorecen la proliferación de infecciones por Enterococo y hongos.

Después de tres años de iniciado el programa de vigilancia se observó un decremento en la tasa de II, el cual se mantuvo hasta 1993, año en que se establece el Programa Prospectivo de Vigilancia de Herida Quirúrgica (figura 1). Consideramos que este incremento en mucho se debe a la captación de infecciones intrahospitalarias de herida quirúrgica y no a una falla del programa de vigilancia y prevención de dichas infecciones, ya que el fenómeno se relaciona principalmente con episodios de infección de HQX (figura 2). Por otra parte, se observa una elevación en la tasa de bacteriemias intrahospitalarias primarias en las neumonías intrahospitalarias; la explicación del fenómeno no es clara, puesto que las últimas prácticamente se triplican. El incremento de la neumonía intrahospitalaria coincide en tiempo con la implementación del servicio de fisioterapia pulmonar, por lo que es necesario realizar un estudio para investigar los factores de riesgo de dicha neumonía en nuestra institución y así establecer medidas preventivas y específicas.

La tendencia hacia el incremento en la tasa II en nuestro hospital puede deberse a varios factores ${ }^{23} \mathrm{en}$ tre ellos, al establecimiento de un sistema de vigilancia con más experiencia que disminuye el subregistro; al incremento en la carga de trabajo; a la crisis económica que produce la disminución en insumos y, por último, a los avances de la tecnología, cuya aplicación incrementa los riesgos de sufrir II (procedimientos invasivos diagnósticos y terapéuticos, catéteres de larga estancia, neutropenia prolongada).

Es notable la ausencia de trabajos que refieren la tasa general de II en los enfermos con cáncer. Los detectados fueron dados a conocer en la década de los ochenta ${ }^{11,12}$ y sólo uno a principios de los noventa. ${ }^{13} \mathrm{Si}$ bien puede parecer lógico que los enfermos con cáncer tengan hoy un mayor riesgo de infectarse, a la fecha no hay estudios que comparen tasas de II en poblaciones con y sin cáncer para sustentar esta afirmación.
La única excepción son los pacientes con leucemia, enfermedad claramente relacionada con los periodos prolongados de neutropenia, factor de riesgo para infección ampliamente reconocido.

\section{Referencias}

1. Dirección General de Estadística e Informática. Mortalidad 1997. México, D.F.: Secretaría de Salud, 1998:69-80.

2. Chanock S. Evolving risk factors for infectious complications of cancer therapy. Hematol O ncol C lin N orth Am 1993;7:771-793.

3. Koll BS, BrownAE. Changing patterns of infections in the immunocompromised patient with cancer. Hematol O ncol Clin N orth Am 1993;7: 753-769.

4. Velasco E, Thuler LC, Martins CA, D ias LM, Gonclaves VM. N osocomial infections in an oncology intensive care unit. Am J Infect Control 1997; 25:458-462

5. Brown AE. N eutropenia, fever and infection. Am J Med 1984;76: 421-428.

6. Center for Disease Control and Prevention. $O$ utline for surveillance and control of nosocomial infections. Atlanta (GA): CDC, US D epartament of Health and Human Services, 1972.

7. Lazo de la Vega-Jasso S,Aranda-C ortez G,Téllez-González P.Vigilancia de infecciones nosocomiales en el paciente con cáncer. Salud Publica Mex 1986;28:636-641

8. Haley RW, Culver DH, W hite JW, Morgan W M, Emori TG. The nation wide infection rate.A new need for vital statistics. Am J Epidemiol 1985; 121:159-168.

9. Ponce de León-Rosales S. Infecciones intrahospitalarias y calidad de la atención médica. ¿Es posible ahorrar en salud? Salud Publica Mex 1991;33: 3-8.

10. Ponce de León-Rosales S, G arcía-García ML,Volkow-Fernández P. Resultados iniciales de un programa de vigilancia de infecciones nosocomiales en los institutos nacionales de salud. Salud Publica Mex 1986;28: 583-592.

11. Rotstein C, Cummings KM, N icolaou AL, Lucey J, Fitzpatrick J. N osocomial infection rates at an oncology center. Infect Control 1988;9:13-19. 12. Robinson GV,Tegtmeier BR, Zaia JA. Brief Report: N osocomial infection rates in a cancer treatment center. Infect Control 1984;5:289-294.

13. Coullioud D, Combe F, Latour JF, Chauin F, Bigot P. Incidence of nosocomial infections in a cancer center; clinical and bacteriological data. Bull Cancer 1990;77:893-900.

14. W hite MC. Mortality associated with nosocomial infections: Analysis of multiple cause-of-death data. J Clin Epidemiol 1993;46:95-100.

15. Pittet D, Harbarth S, Ruef C, Francioli P, Sudre P, Pétigant et al. Prevalence and risk factors for nosocomial infections in four University Hospitals in Switzerland. Infect Control Hosp Epidemiol 1999;20:37-42.

16.Vilar-Compte D, Mohar A, Sandoval S, Gordillo P, De la Rosa M,Volkow $P$. Surgical site infections at the $N$ ational Institute in Mexico. A case control study. Am J Infect Control 2000;28:14-20.

17. Haley RW, Culver DH, Emori TG et al. Progress report on the evaluation of the efficacy of infection survelliance $\&$ control programs. En: Dixon RL, ed. N osocomial Infection. N ueva York (NY):York Medical Books, 1981.

18. Vilar-Compte D, Sandoval S, Gordillo P, de la Rosa M, Sánchez-Mejorada $G$, Volkow P.Vigilancia de las infecciones de herida quirúrgica. Experiencia de 18 meses en el Instituto $\mathrm{N}$ acional de $\mathrm{C}$ ancerología. Salud Publica Mex 1999;41supl1:44-50. 
19. Martone W J, Jarvis W R, C ulver DH, Haley RW. Incidence and nature of endemic and epidemic nosocomial infections. En: Bennett JV, Brachman PS, ed. Hospital infections. 3a. edición. Boston (MA): Little Brown, 1992:577-596.

20. Berghmans T, Crokaert F, Markiewicz E, Sculier JP. Epidemiology in the adult medical intensive care unit of a cancer hospital. Support $\mathrm{C}$ are $\mathrm{C}$ ancer 1997;5:234-240.

21. Greene JN . Catheter-related complications of cancer therapy. Infect $D$ is Clin N orth Am 1996;10:255-295.
22.W ey S. N osocomial infection in the compromised host. En:W enzel R, ed. Prevention and control of nosocomial infections. 2a. edición. Baltimore (MD):W illiams and W ilkins, 1993:923-957.

23. Dixo RE. Investigation of endemic and epidemic nosocomial infections. En: Bennett JV, Brachman PS, ed. Hospital Infections. 3a. edición. Boston (MA): Little Brown, 1992:109-133. 